\title{
A DNA Microarray System for Analyzing Complex DNA Samples Using Two-color Fluorescent Probe Hybridization
}

\author{
Dari Shalon, ${ }^{1,4}$ Stephen J. Smith, ${ }^{3}$ and Patrick O. Brown ${ }^{1,2,5}$ \\ ${ }^{1}$ Howard Hughes Medical Institute and Departments of ${ }^{2}$ Biochemistry and ${ }^{3}$ Molecular and Cellular \\ Physiology, Stanford University, Stanford, California 94305
}

\begin{abstract}
Detecting and determining the relative abundance of diverse individual sequences in complex DNA samples is a recurring experimental challenge in analyzing genomes. We describe a general experimental approach to this problem, using microscopic arrays of DNA fragments on glass substrates for differential hybridization analysis of fluorescently labeled DNA samples. To test the system, 864 physically mapped $\lambda$ clones of yeast genomic DNA, together representing $>75 \%$ of the yeast genome, were arranged into $1.8-\mathrm{cm} \times 1.8-\mathrm{cm}$ arrays, each containing a total of 1744 elements. The microarrays were characterized by simultaneous hybridization of two different sets of isolated yeast chromosomes labeled with two different fluorophores. A laser fluorescent scanner was used to detect the hybridization signals from the two fluorophores. The results demonstrate the utility of DNA microarrays in the analysis of complex DNA samples. This system should find numerous applications in genome-wide genetic mapping, physical mapping, and gene expression studies.
\end{abstract}

Many problems in genome analysis depend on determining what specific sequences are represented in a complex DNA or RNA sample and at what abundance, for example, what genes are represented in a specific chromosome band or YAC clone, what intervals are amplified or deleted in a particular cancer cell, or what genes are expressed in specific cells under specific conditions. As a general approach to this problem, we have developed a system for making microarrays of DNA samples on glass substrates, probing them by hybridization with complex fluorescentlabeled probes, and using a laser-scanning microscope to detect the fluorescent signals representing hybridization. Fluorescent labeling allows for simultaneous hybridization and separate detection of the hybridization signal from two or more probes. This in turn allows very accurate and reliable measurement of the relative abundance of specific sequences in two complex samples.

\section{RESULTS}

\section{Array Hybridization Pattern}

Figure 1 shows the two-color fluorescent scan of a yeast genomic array following hybridization

\footnotetext{
4Present address: Synteni, Inc., Palo Alto, California 94305.

${ }^{5}$ Corresponding author.

E-MAll pbrown@cmgm.stanford.edu, http://cmgm. stanford.edu/pbrown; FAX (415) 723-1399.
}

with a mixed probe consisting of lissaminelabeled DNA from the 6 largest yeast chromosomes together with fluorescein-labeled DNA from the 10 smallest yeast chromosomes. A red color indicates that yeast sequences present in the lissamine-labeled hybridization probe hybridized to an array element. A yellow-green color indicates that yeast sequences present in the fluorescein-labeled hybridization probe hybridized to an array element. An orange color indicates cross-hybridization of both chromosome pools to an array element (e.g., dispersed repetitive elements, such as Ty1 elements).

Each clone was spotted twice, resulting in duplicate hybridization patterns in adjacent quadrants of the array. Control DNA spots, which were randomly amplified in the same manner as the $\lambda$ clone array elements, are located in the bottom corner of each quadrant. " $\mathrm{A}$ " points to a pair of spots containing total yeast genomic DNA. These spots appear orange because both chromosome pools hybridized to yeast genomic DNA. The negative controls are as follows: " $\mathrm{B}$ " points to a pair of spots of wild-type $\lambda \mathrm{DNA}$, "C" points to a pair of human genomic DNA spots, and " $\mathrm{D}$ " points to a pair of $\phi X 174$ DNA spots. The lack of a hybridization signal at these three negative control spots indicates that the hybridization was specific for yeast sequences. 


\section{SHALON ET AL.}

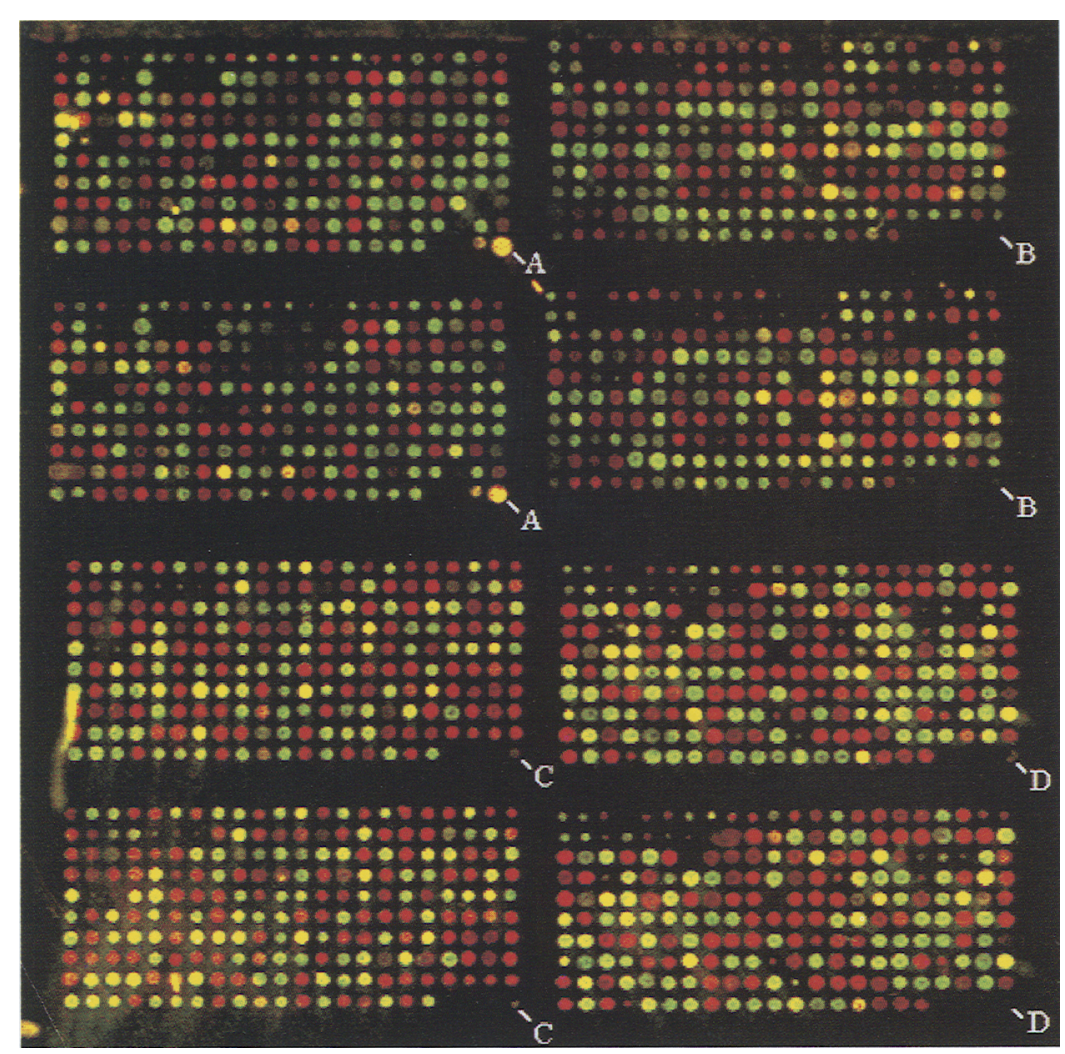

Figure 1 Two-color fluorescent scan of a 1.8-cm $\times 1.8-\mathrm{cm}$ yeast array of $\lambda$ clones of yeast genomic DNA. The DNA spots are spaced at a distance of $380 \mu \mathrm{m}$ from center to center. A probe mixture consisting of DNA from the 6 largest yeast chromosomes $(4,7,12,13,15,16)$ labeled with lissamine (red dots) and DNA from the 10 smallest yeast chromosomes $(1,2,3,5,6,8,9,10,11,14)$ labeled with fluorescein (yellowgreen dots) was hybridized to the array. A pair of yeast genomic DNA spots $(A)$ served as a positive control. The three negative controls are $\lambda$ DNA $(B)$, human genomic DNA $(C)$, and $\phi \times 174$ DNA $(D)$.

\section{Karyotype Depiction of the Array Hybridization Pattern}

The inserts contained in the arrayed $\lambda$ clones have been mapped physically (Riles et al. 1993). The clones are arrayed in a random but known order on the array. Therefore, using the identity of each clone along with its physical map information, the pattern of hybridization to the yeast array can be represented in the form of a karyotype of the yeast genome, as shown in Figure 2. The color of any segment of the ideogram representing an individual chromosome on the karyotype is directly determined by the ratio of red and green hybridization signals at the array positions of the corresponding clones. The lengths of the discrete colored segments of each chromosome correspond to the physical lengths of the yeast inserts. The chromosome segments colored black represent either intervals of the genome that are not represented by clones in the library $(90 \%)$ or false-negative hybridization signals on the array (10\%). Most of these false negatives are attributable to failures of the PCR amplification of the $\lambda$ clones, though occasional failures of the arraying process or nonuniform surface preparation could account for a small fraction of the false-negative signals. The large gap on chromosome 12 is the region coding for ribosomal DNA that was not represented among the arrayed clones. Genomic intervals represented by overlapping clones were assigned a color based on the hybridization signals of only one of the overlapping clones, chosen at random.

Note that in this representation of a yeast karyotype, the largest six chromosomes are mainly colored red. This indicates that most of the arrayed clones that were mapped previously to these six large chromosomes hybridized primarily to the lissamine-labeled probe prepared from the corresponding purified chromosomes. Conversely, the smallest 10 chromosomes are mainly colored green in this image, matching the original CHEF gel isolation of the chromosomes used as the hybridization probe. The experiment was repeated with the yeast genome split into six discrete chromosome pools containing 2-4 chromosomes per pool using CHEF gel electrophoresis. The chromosomes in each pool were extracted from the gel, amplified, and fluorescently labeled. The six chromosome pools were hybridized to six separate yeast arrays. Forty-four $\lambda$ clones gave a positive hybridization signal on all six arrays indicating that they contain yeast repetitive sequences (data not shown). These 44 clones and 10 clones with very weak hybridization signals were not included in the data set used to produce this karyotype.

There were $\sim 40$ anomalous clones, which appear in this karyotype representation as green bands on the otherwise red chromosomes or red 
DNA MICROARRAYS FOR ANALYZING COMPLEX DNA SAMPLES

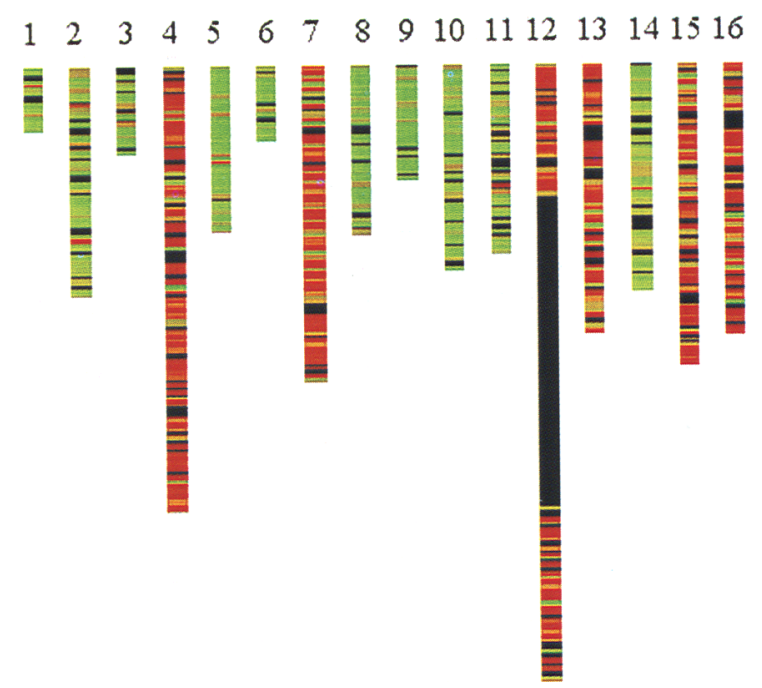

Figure 2 Computer-generated ideogram representing a karyotype of $S$. cerevisiae, based on the normalized hybridization signals from the array shown in Fig. 1. Note that the 6 largest chromosomes are mainly red and the 10 smallest chromosomes are mainly green. Black stripes represent intervals not represented by clones in the array or for which the corresponding clones gave false-negative hybridization signals.

bands on the otherwise green chromosomes. Four randomly chosen examples of these anomalous clones were analyzed by hybridizing the clones to vertical strips cut from a Southern blot of CHEF gel-separated yeast chromosomes. In each case, the hybridization patterns of the anomalous clones corroborated the chromosomal locations assigned by the microarray hybridization results (data not shown). Two clones that were thought to map to the 10 smallest chromosomes were found to hybridize preferentially to the probe representing the 6 largest chromosomes and thus appear as anomalous red bands on the karyotype. Both hybridized to one of the six largest chromosomes on the Southern blot. Similarly, two clones that appear as anomalous green bands on the karyotype were found to hybridize to one of the 10 smallest chromosomes on the Southern blot. Thus, the anomalous clones are probably the result of sample tracking errors or, possibly, of errors in the published restrictiondigest-based physical map on which the karyotype representation was based (Riles et al. 1993).

\section{DISCUSSION}

The DNA microarray hybridization system reported here is conceptually and functionally similar to fluorescent in situ hybridization (FISH) to metaphase chromosomes, with three important differences. First, the target elements of the microarrays can, in principle, be any length or composition, from megabase YAC clones or microdissected chromosome bands to individual cDNA clones, to short oligonucleotides. This versatility allows the user to choose characteristics, such as the mapping resolution and genetic complexity of each array element, to suit a particular application. Second, the hybridization signals are localized to discrete elements of known size and location, making them easier to identify and quantitate than the hybridization signals from irregularly shaped metaphase spreads. Third, microarrays are more consistent and potentially amenable to automated production, hybridization, and data analysis than metaphase spreads.

Arrays of DNA samples on porous membranes, for example, dot blots, have long been used as a basic tool in molecular biology. Dotblot membranes are usually at least $8 \times 12 \mathrm{~cm}$ in size, require the use of milliliter volumes of hybridization solution, and are limited, owing to autofluorescence and scattering, to radioactive, chemiluminescent, and colorimetric hybridization detection methods (Ross et al. 1992). Microarrays made on glass surfaces, on the other hand, can be mass-produced and are comparatively inexpensive, convenient, and compatible with fluorescent hybridization detection methods. Furthermore, a glass surface, when appropriately treated, has very low nonspecific binding of labeled hybridization probes, resulting in lower backgrounds than are encountered typically with porous membranes. For hybridizations with very complex probes, the concentration of the labeled probe DNA is a limiting factor in the sensitivity of the assay. Minimizing the volume of the probe solution in a hybridization, by restricting the target to a small area and by using a nonporous substrate, makes it practical to achieve very high probe concentrations.

One important advantage of fluorescently labeled probes is that, unlike most radioactive and chemiluminescent signals, fluorescent signals do not disperse and therefore allow for very dense array spacing. A unique, and probably the most important, advantage of fluorescent probes is that the hybridization signals from two or more differently labeled probes hybridized to the same target element can be detected separately. In this way, two-color hybridization detection allows for a direct and quantitative comparison of the 


\section{SHALON ET AL.}

abundance of specific sequences between two probe mixtures that are hybridized competitively to a single array. The absolute intensity of a hybridization signal at a particular element in an array can vary owing to experimental factors such as variations in the amount of DNA deposited on the array, variations in the hybridization or wash conditions between experiments, or variations in the hybridization characteristics of the different DNA sequences on the array. The ratio of the two signals at any element in an array, however, is relatively insensitive to these confounding factors because they affect both probe mixtures equivalently. This ratio therefore accurately reflects the relative abundance of the cognate sequence in the two probe samples. This is the principle underlying the technique of comparative genomic hybridization (CGH), which is used to detect changes in the copy number of specific chromosomes or chromosomal regions (Kallioniemi et al. 1992). CGH is based on measuring the relative fluorescent hybridization intensities of two genomic-complexity hybridization probes, for example, probes representing genomic DNA from normal and affected tissue samples, which are labeled with two distinct fluorophores and hybridized simultaneously to a metaphase spread. DNA microarray representations of the human genome may provide a more convenient and higher resolution alternative to metaphase chromosomes for CGH.

Cross-hybridization between related sequences is an important problem faced by any hybridization-based assay, including the DNA microarray assay described here. Studies are now in progress to quantitate the extent of crosshybridization between related sequences of varying homology and length, in DNA microarray hybridizations. The stringency of hybridization and washing can be controlled by varying the salt concentration and temperature as in conventional membrane-based hybridizations. Crosshybridization caused by repetitive sequences can be minimized by prehybridization of the probe or array with vast excess of unlabeled copies of the repetitive sequences.

Alternative methods have been described for making microarrays of very short DNA sequences, involving photolithography (Pease et al. 1994) or physical masking (Maskos and Southern 1992) methods. These in situ synthesis methods are inherently limited to low complexity array elements consisting of oligonucleotides. For complex-probe hybridizations, the specificity of hybridization is improved by using DNA fragments substantially longer than oligonucleotides. Moreover, the in situ synthesis approaches to array fabrication depend on prior knowledge of the sequence to be recognized by each array element. The approach described here makes microarrays by transferring tiny volumes of DNA samples from microwell storage plates to a solid substrate. Thus, nucleic acids (or other molecules) of virtually any length or any origin can be arrayed, and knowledge of their sequences is not required.

The arrays used in these experiments do not represent the maximal achievable density of elements. We have found that the spacing between the spots can be decreased by shrinking the contact area of the printing tip and by increasing the hydrophobicity of the glass surface. Microarrays with $100-\mu \mathrm{m}$ feature size have been tested successfully in pilot experiments (data not shown). Assuming the projected availability of the appropriate physically mapped human genomic clones (Hudson et al. 1995), arrays at $100-\mu \mathrm{m}$ spacing would allow for 10,000 discrete intervals of the human genome to be represented in a $1-\mathrm{cm}^{2}$ array. Such an array could be used for mapping at a resolution of $<0.5 \mathrm{Mb}$. Experiments are in progress to explore the feasibility of such arrays.

Our initial motivation for developing these microarrays arose from the need for abundant and inexpensive genomic arrays for genomic mismatch scanning (GMS) (Nelson et al. 1993), a method of genetic linkage analysis based on identification of the regions of "identity by descent" between affected relative pairs using a single complex-probe hybridization to an array of genomic clones. Experiments using these arrays to map quantitative trait loci in yeast by GMS are currently in progress (J. deRisi, D. Lashkari, L. Penland, L. McAllister, J. McCusker, R. Davis, and P.O. Brown, unpubl.).

Microarrays of cDNA clones, prepared using the system described here, have been used for quantitative monitoring of gene expression patterns in Arabidopsis (Schena et al. 1995), S. cerevisiae (D. Lashkari, J. deRisi, L. Penland, P.O. Brown, and R. Davis, unpubl.), and human tissues (J. deRisi, M. Bittner, P. Meltzer, L. Penland, J. Trent, and and P.O. Brown, unpubl.). We anticipate that DNA microarrays of the kind described here will be useful in additional applications for which conventional dot blots, highdensity gridded arrays on porous membranes, or FISH are currently used. These potential applica- 


\section{DNA MICROARRAYS FOR ANALYZING COMPLEX DNA SAMPLES}

tions include comparative genomic hybridization (Kallioniemi et al. 1992), sequencing by hybridization (Drmanac et al. 1993), physical mapping of cloned or amplified sequences (Billings et al. 1991), and economical distribution of reagents for integrated genetic and physical mapping based on a common set of arrayed clones (Zehetner and Lehrach 1994).

\section{METHODS}

\section{Amplification of Target DNA Elements}

The array elements were prepared from physically mapped $\lambda$ clones (Riles et al. 1993). The $\lambda$ clones were amplified using randomly primed polymerase chain reaction (PCR) based on published and unpublished protocols (Bohlander et al. 1992; S. Nelson, unpubl.). The phage lysates were amplified in a 10- $\mu$ l PCR reaction using $5 \mu \mathrm{M}$ final concentration of primer A (GCTATCTTCAAGATCANNNNNN), $200 \mu \mathrm{M}$ dNTPs, and 1 unit of Taq polymerase. Round A consisted of five cycles at $94^{\circ} \mathrm{C}$ for $1 \mathrm{~min}, 25^{\circ} \mathrm{C}$ for $1.5 \mathrm{~min}$, $25-72^{\circ} \mathrm{C}$ over $7 \mathrm{~min}$, and $72^{\circ} \mathrm{C}$ for $3 \mathrm{~min}$ using Taq polymerase (BMB). For round $B$, the reaction volume was brought up to $100 \mu \mathrm{l}$ for a final concentration of $2 \mu \mathrm{M}$ of primer B (GCTATCTTCAAGATCA), $200 \mu \mathrm{M}$ dNTPs, and 4 units of Taq polymerase. Round B consisted of 30 cycles of $94^{\circ} \mathrm{C}$ for $1 \mathrm{~min}, 56^{\circ} \mathrm{C}$ for $2 \mathrm{~min}$, and $72^{\circ} \mathrm{C}$ for $3 \mathrm{~min}$. The amplification was performed in 96-well plates using crude phage lysates as the templates, resulting in an amplification of both the $35-\mathrm{kb} \lambda$ vector and the $5-\mathrm{kb}$ to $15-\mathrm{kb}$ yeast insert sequences as a distribution of PCR products between $250 \mathrm{bp}$ and $1500 \mathrm{bp}$ in length.

The PCR products were purified and transferred into TE (10 mm Tris, 1 mm EDTA at pH 8.0) buffer using Sephadex G50 gel filtration (Pharmacia) and evaporated to dryness at room temperature overnight. Each of the 864 am-

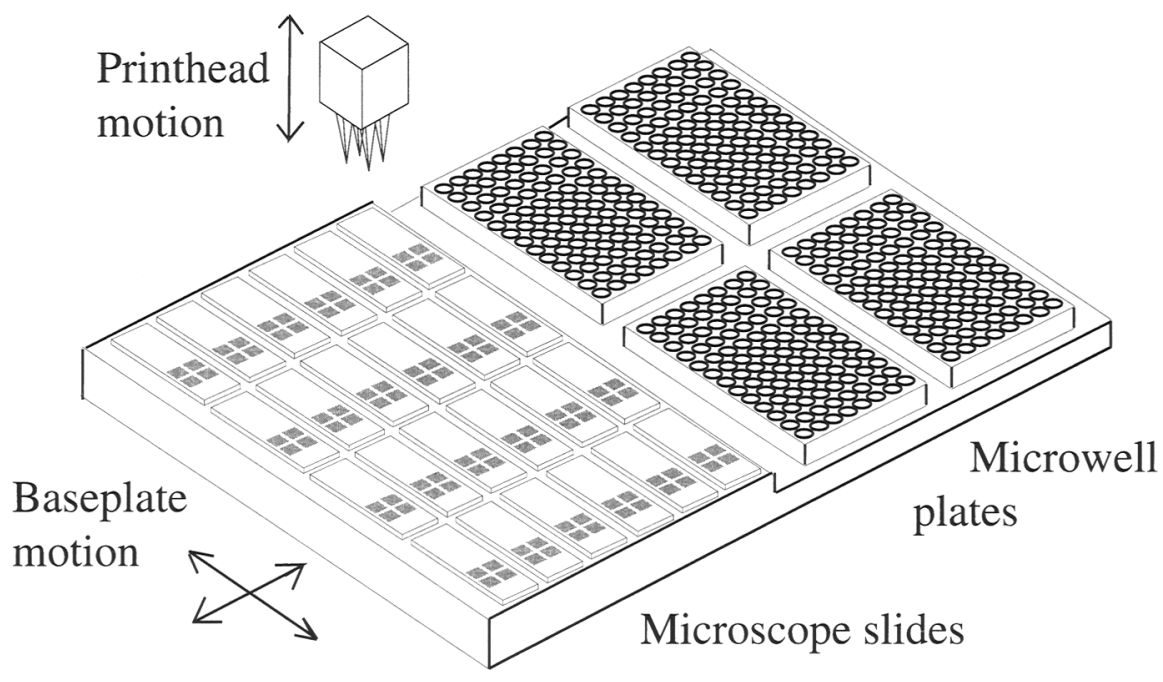

Figure 3 The layout of the arraying machine. All motions are under computer control. For more details of the arraying machine, see web page http:// cmgm.stanford.edu/pbrown. plified $\lambda$ clones was rehydrated in $15 \mu \mathrm{l}$ of $3 \times$ SSC $\left(20 \times\right.$ SSC $=3 \mathrm{M} \mathrm{NaCl}, 0.3 \mathrm{M} \mathrm{Na}_{3}$ citrate $)$ in preparation for spotting onto the glass under normal room temperature conditions.

\section{Preparation of DNA Microarrays}

The microarrays were fabricated on poly-L-lysine coated microscope slides (Sigma). A custom-built arraying machine, consisting of four tweezer-like printing tips mounted $9 \mathrm{~mm}$ apart on a computer-controlled robotic stage (Shalon 1996), loaded $1 \mu \mathrm{l}$ of the concentrated PCR product directly from corresponding clusters of four wells of 96-well storage plates and deposited $\sim 5 \mathrm{nl}$ of each sample onto each of 40 slides. Surface tension loaded the sample into the printing tip directly from the microwell plate and held the sample in the tip during the printing operation. Printing was achieved by lightly tapping the tip against the glass surface. The open-capillary design allowed for rapid rinsing and drying of the tips between samples. Figure 3 shows the layout of the arraying machine. Figure 4 shows a detailed view of the four printing tips and the staggered printing pattern on the microscope slides. Adjacent samples were spotted $380 \mu \mathrm{m}$ apart on the slides. After each set of four samples was printed onto 40 slides, the printing tips were rinsed with a jet of water for $2 \mathrm{sec}$ and then dried by lowering the tips onto a sponge for 2 sec. The process was repeated for all 864 samples and eight control spots.

After the spotting operation was complete, the slides were rehydrated in a humid chamber at room temperature for $2 \mathrm{hr}$, baked in an $80^{\circ} \mathrm{C}$ vacuum oven for $2 \mathrm{hr}$, then rinsed in $0.1 \%$ sodium dodecyl sulfate (SDS) to remove unadsorbed DNA. To reduce nonspecific adsorption of the labeled hybridization probe to the poly-L-lysine coated glass surface, the slides were treated with succinic anhydride. One gram of succinic anhydride was dissolved in $100 \mathrm{ml}$ of 1 -methyl-2-pyrrolidinone and then $100 \mathrm{ml}$ of $0.2 \mathrm{M}$ boric acid ( $\mathrm{pH} 8.0$ ) was added. The arrays were soaked in this solution for $10 \mathrm{~min}$ and then rinsed in distilled water four times for $5 \mathrm{~min}$ each. Immediately before use, the arrayed DNA elements were denatured by placing the slide in distilled water at $90^{\circ} \mathrm{C}$ for $2 \mathrm{~min}$.

\section{Amplification and Labeling of Hybridization Probe}

The 16 chromosomes of Saccharomyces cerevisiae were separated using a contour-clamped homogeneous electric field (CHEF) agarose gel apparatus (Bio-Rad) (Chu et al. 1986). The 6 largest chromosomes were isolated in one gel slice and the smallest ten chromosomes in a second gel slice. The DNA from each slice was recovered using a gel extraction kit 


\section{SHALON ET AL.}

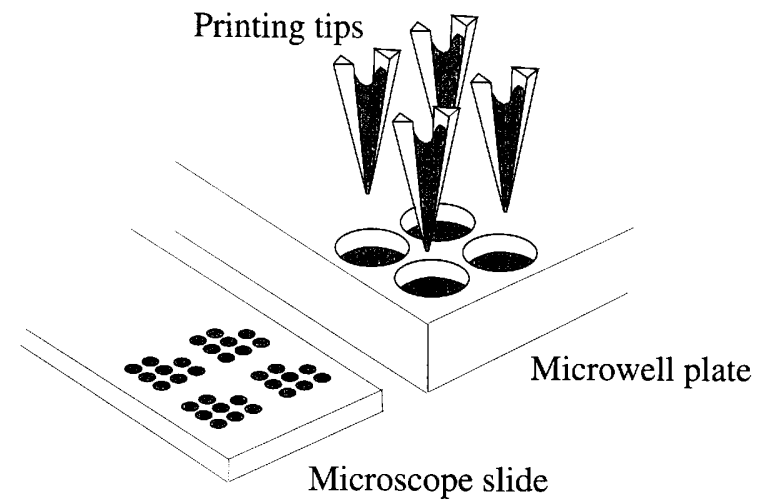

Figure 4 A close-up view of the four opencapillary printing tips. The tips are $9 \mathrm{~mm}$ apart and fit into four adjacent wells of a standard microwell plate and print arrays in a staggered fashion on microscope slides. For more details of the printing tips, see web page http://cmgm.stanford.edu/pbrown.

(Qiagen) and randomly amplified in a manner similar to that used in amplifying the target $\lambda$ clones (Grothues et al. 1993). The main difference between this amplification procedure and the one used for the $\lambda$ array elements is a filtration step between rounds A and B to remove primerdimers and the use of a random 9-mer 3' end on primer A. Following amplification, $2.5 \mu \mathrm{g}$ of each of the amplified chromosome pools were separately random-primer labeled using Klenow polymerase (Amersham) with a lissamineconjugated nucleotide analog (DuPont NEN) for the pool containing the 6 largest chromosomes and with a fluorescein-conjugated nucleotide analog (BMB) for the pool containing the smallest 10 chromosomes. The two fluorescent-labeled pools were mixed and concentrated using an ultrafiltration device (Amicon).

\section{Hybridization}

Five micrograms of the hybridization probe, consisting of both chromosome pools in $7.5 \mu \mathrm{l}$ of TE, was denatured in a boiling water bath and then snap-cooled on ice. Concentrated hybridization solution $(2.5 \mu \mathrm{l})$ was added to a final concentration of $5 \times \mathrm{SSC} / 0.1 \%$ SDS. The entire $10 \mu \mathrm{l}$ of probe solution was transferred to the array surface, covered with a coverslip, placed in a custom-built single-slide humidity chamber, and incubated in a $60^{\circ} \mathrm{C}$ water bath for 12 hr. The custom-built waterproof slide chamber has a cavity just slightly bigger than a microscope slide and was kept at $100 \%$ humidity internally by the addition of $2 \mu \mathrm{l}$ of water in a corner of the chamber. The slide was rinsed in $5 \times$ $\mathrm{SSC} / 0.1 \% \mathrm{SDS}$ for $5 \mathrm{~min}$ and then in $0.2 \times \mathrm{SSC} / 0.1 \%$ SDS for $5 \mathrm{~min}$. All rinses were at room temperature. The array was then air dried, and a drop of antifade (Molecular Probes) was applied to the array under a $24-\mathrm{mm} \times 30-\mathrm{mm}$ coverslip in preparation for scanning.

\section{Detection and Analysis}

A custom-built laser scanner was used to detect the two- color fluorescence hybridization signals from 1.8 $\mathrm{cm} \times 1.8-\mathrm{cm}$ arrays at $20-\mu \mathrm{m}$ resolution. The glass substrate slide was mounted on a computer-controlled, twoaxis translation stage (PM-500, Newport, Irvine, CA) that scanned the array over an upward-facing microscope objective $(20 \times, 0.75 \mathrm{NA}$ Fluor, Nikon, Melville, NY) in a bidirectional raster pattern. A water-cooled Argon/Krypton laser (Innova 70 Spectrum, Coherent, Palo Alto, CA), operated in multiline mode, allowed for simultaneous specimen illumination at $488.0 \mathrm{~nm}$ and $568.2 \mathrm{~nm}$. These two lines were isolated by a $488 / 568$ dual-band excitation filter (Chroma Technology, Brattleboro, VT). An epifluorescence configuration with a dual-band $488 / 568$ primary beam splitter (Chroma) excited both fluorophores simultaneously and directed fluorescence emissions toward the two-channel detector. Emissions were split by a secondary dichroic mirror with a 565 transition wavelength onto two multialkali cathode photomultiplier tubes (PMT; R928, Hamamatsu, Bridgewater, NJ), one with an HQ535/50 bandpass barrier filter and the other with a D630/60 bandpass barrier filter (Chroma). Preamplified PMT signals were read into a personal computer using a 12-bit analog-todigital conversion board (RTI-834, Analog Devices, Norwood, MA), displayed in a graphics window, and stored to disk for further rendering and analysis. The back aperture of the $20 \times$ objective was deliberately underfilled by the illuminating laser beam to produce a large-diameter illuminating spot at the specimen $(5-\mu \mathrm{m}$ to $10-\mu \mathrm{m}$ halfwidth). Stage scanning velocity was $100 \mathrm{~mm} / \mathrm{sec}$, and PMT signals were digitized at $100 \mu \mathrm{sec}$ intervals. Two successive readings were summed for each pixel, such that pixel spacing in the final image was $20 \mu \mathrm{m}$. Beam power at the specimen was $\sim 5 \mathrm{~mW}$ for each of the two lines.

The scanned image was despeckled using a graphics program (Hijaak Graphics Suite) and then analyzed using a custom image gridding program that created a spreadsheet of the average red and green hybridization intensities for each spot. The red and green hybridization intensities were corrected for optical cross talk between the fluorescein and lissamine channels, using experimentally determined coefficients.

\section{ACKNOWLEDGMENTS}

This research was supported by grant HG00450 from the National Institutes of Health-National Center for Human Genome Research, a National Science Foundation graduate fellowship to D.S., and by the Howard Hughes Medical Institute. P.O.B. is an assistant investigator of the Howard Hughes Medical Institute. We thank John Mulligan and John McCusker for help in preparing and amplifying the $\lambda$ clones used in the arrays, Ren Xin Xia for writing the scanner control software and the image gridding and automatic karyotyping programs, Jeff van Ness at Darwin Molecular Corporation for suggesting the use of succinic anhydride, Stan Nelson, Linda McAllister, Joe deRisi, and Lolita Penland for helpful suggestions in the course of this work, and Joe deRisi and Linda McAllister for helpful comments on the manuscript.

The publication costs of this article were defrayed in part by payment of page charges. This article must therefore be hereby marked "advertisement" in accordance with 18 USC section 1734 solely to indicate this fact. 


\section{DNA MICROARRAYS FOR ANALYZING COMPLEX DNA SAMPLES}

\section{REFERENCES}

Billings, P.R., C.L. Smith, and C.R. Cantor. 1991. New techniques for physical mapping of the human genome. FASEB J. 5: 28-34.

Bohlander, S.K., R. Espinosa III, M.M. LeBeau, J.D. Rowley, and M.O. Diaz. 1992. A method for the rapid sequence-independent amplification of microdissected chromosomal material. Genomics 13: 1322-1324.

Chu, G., D. Vollrath, and R. Davis. 1986. Separation of large DNA molecules by contour clamped homogeneous electric fields. Science 234: 1582-1585.

Drmanac, R., S. Drmanac, Z. Strezoska, T. Paunesku, I. Labat, M. Zeremski, J. Snoddy, W.K. Funkhouser, B. Koop, L. Hood, et al. 1993. DNA sequence determination by hybridization: A strategy for efficient large-scale sequencing. Science 260: 1649-1652.

Grothues, D., C.R. Cantor, and C.L. Smith. 1993. PCR amplification of megabase DNA with tagged random primers (T-PCR). Nucleic Acids Res. 21: 1321-1322.

Hudson, T.J., L.D. Stein, S.S. Gerety, J. Ma, A.B. Castle, J. Silva, D.K. Slonim, R. Baptista, L. Kruglyak, S.H. Xu, et al. 1995. An STS-based map of the human genome. Science 270: 1945-1954.

Kallioniemi, A., O.P. Kallioniemi, D. Sudar, D. Rutovitz, J.W. Gray, F. Waldman, and D. Pinkel. 1992. Comparative genomic hybridization for molecular cytogenetic analysis of solid tumors. Science 258: $818-821$.

Maskos, U. and E.M. Southern. 1992. Parallel analysis of oligodeoxyribonucleotide (oligonucleotide) interactions. I. Analysis of factors influencing oligonucleotide duplex formation. Nucleic Acids Res. 20: 1675-1678.

Nelson, S.F., J.H. McCusker, M. Sander, Y. Kee, P. Modrich, and P.O. Brown. 1995. Genomic mismatch scanning: A new approach to genetic linkage mapping, Nature Genet. 4:11-17.

Pease, A.C., D. Solas, E.J. Sullivan, M.T. Cronin, C.P. Holmes, and S.P. Fodor. 1994. Light-generated oligonucleotide arrays for rapid DNA sequence analysis. Proc. Natl. Acad. Sci. 91: 5022-5026.

Riles, L., J.E. Dutchik, A. Baktha, B.K. McCauley, E.C. Thayer, M.P. Leckie, V.V. Braden, J.E. Depke, and M.V. Olson. 1993. Physical maps of the six smallest chromosomes of Saccharomyces cerevisiae at a resolution of 2.6 kilobase pairs. Genetics 134: $81-150$.

Ross, M.T., J.D. Hoheisel, A.P. Monaco, Z. Larin, G. Zehetner, and H. Lehrach. 1992. High density gridded YAC filters: Their potential as genome mapping tools. In Techniques for the analysis of complex genomes (ed. Rakesh Anand), pp. 137-153. Academic Press, London, UK.

Schena, M., D. Shalon, R.W. Davis, and P.O. Brown. 1995. Quantitative monitoring of gene expression patterns with a complementary DNA microarray. Science 270: $467-470$.

Shalon, D., 1995. "DNA micro arrays: A new tool for genetic analysis." Ph.D. thesis, Stanford University, Stanford, CA.

Zehetner, G. and H. Lehrach. 1994. The reference library system-Sharing biological material and experimental data. Nature 367: 489-491.

Received March 4, 1996; accepted in revised form May 9, 1996. 


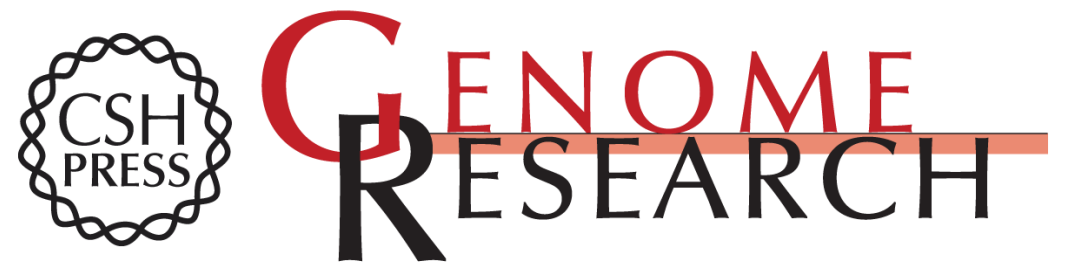

\section{A DNA microarray system for analyzing complex DNA samples using two-color fluorescent probe hybridization.}

D Shalon, S J Smith and P O Brown

Genome Res. 1996 6: 639-645

Access the most recent version at doi:10.1101/gr.6.7.639

References This article cites 13 articles, 7 of which can be accessed free at:

http://genome.cshlp.org/content/6/7/639.full.html\#ref-list-1

\section{License}

Email Alerting Receive free email alerts when new articles cite this article - sign up in the box at the Service top right corner of the article or click here.

\section{Affordable, Accurate Sequencing.}

To subscribe to Genome Research go to:

https://genome.cshlp.org/subscriptions 